\title{
LSD, Caffeine and Cholera: Possible Causes of Schizophrenia
}

Paul TE Cusack*

1641 Sandy Point Rd, Saint John, NB, E2K 5E8, Canada

\begin{abstract}
Schizophrenia $(\mathrm{Sz})$ hospitalization is on the rise for young men. There is a link between caffeine and calcium absorption. Calcium is necessary for a healthy nervous system. In this brief paper, I show that the cause of Sz increase among young men may be because of the dramatic increase of caffeine intake (Coffee) among that group. In addition, use of hallucinogens (LSD) among young men may be the sole explanation for the rise in Sz among that group. We also examine the connection between cholera and Schizophrenia. In this paper, we suggest possible causes of $\mathrm{Sz}$ that may warrant closer examination by researchers.
\end{abstract}

Keywords: Schizophrenia; Caffeine; LSD; Calcium; Ferric chloride disease; Cholera; Adenine

\section{Introduction}

In this brief communication, I provide an explanation for the rise of Sz hospitalizations among young men (20-29). First, I consider a previous paper that examined the environmental reasons for the cause of $\mathrm{Sz}$, namely hyper-ferric chloride. In another paper, I examined the possible cause of $\mathrm{Sz}$ resulting from a vitamin $\mathrm{D}$ deficiency and associated calcium deficiency, necessary for a normal nervous system functioning. Finally, I consider the implications from LSD use. In fact, mathematically, this could provide the sole reason for? $\mathrm{Sz}$ in in hospitalization rates increase of $28 \%$ among young men.

\section{Hyper Ferric-Chloride Disease and Schizophrenia}

\section{Hyper caffeine and Schizophrenia}

Consider the result form the paper by the author for the Ferric chloride disease. Vitamin D is necessary to avoid depression. Tryptophan combined with cofactor iron breaks down leaving the brain short of serotonin. Calcium in milk is taken up in $\mathrm{CaCl}_{2}$ which was need for nervous system, development. The same is with $(\mathrm{K})$ Potassium. Vitamin C Causes Iron not to be absorbed. It stays in the blood. Too High Iron levels are bad for the nervous system. This all causes a deformed nervous system. Out ward signs are red hair (or blonde), large head, blue eyes, large genitals, hyper and hypo thyroidism, high blood pressure, color blindness, obnoxious aggressive personality. Results Nervous disorders including Sz, Parkinson's, Alzheimer's, Infertility, Mental Retardation, Depression, etc. [1].

I considered that, with the marked increase in caffeine (coffee) consumption in Canada, for example, perhaps there is a link between Schizophrenia and caffeine. There may well be.

We know that calcium is necessary for normal nervous system functioning. Vitamin D ha be cited as a possible cause for Sz. Caffeine affects the calcium uptake in the body.

Another impact of caffeine that is particularly important for adolescents is its relationship to bone health. It has been reported that individuals who consume a high amount of caffeine may have bones with less density, ultimately leading to weaker bones. This happens because caffeinated beverages may interfere with your body's ability to absorb calcium [2].

Caffeine may well have a negative impact on the nervous system functioning, but there is a more prevalent cause that explains the whole increase seen in hospitalizations form Sz in young men, namely, LSD.

Between 1987 and 1999, hospitalizations for schizophrenia increased slightly among women (3\%), but they increased dramatically among men (28\%) (Figure 1) [3].

Such psychedelics are more commonly consigned to the freewheeling 1960s, when they gained notoriety as part of the countercultural movement. But interest in LSD and other hallucinogenics has grown in recent years, particularly among 20- to 29-year-olds.

\section{Hallucinogen LSD and Schizophrenia}

Young men born in the late 1960's -the Generation "X"- could have been exposed to LSD as kids. In addition, that age group-20-29 is taking hallucinogen is on the increase.

As explained in a previous paper, we see from $\mathrm{Sz}$ and its Cause, (Ob. Cit.), that LSD can theoretically lead to Sz symptoms and Psychosis. Let's look at some calculations and chemistry.

\section{LSD and Schizophrenia}

The $\mathrm{NaOH}$ in those living in a marine environment reacts with the $\mathrm{H}$ on the 8-position forming $\mathrm{NaCl}$ and $\mathrm{HOH}$. The $\mathrm{HCL}$ and Iron in the blood strip the $\mathrm{NH}_{2}$ off of the DNA molecule. This is why LSD induces Schizophrenia.

$$
\begin{aligned}
& \mathrm{NaOH}+\mathrm{LSD}+\mathrm{FeCl}==>\mathrm{NaCl}+\mathrm{H}_{2} \mathrm{O} \\
& \mathrm{Fe}+\mathrm{HCl}==>\text { strips } \mathrm{NH}_{2} \text { off benzene ring on A-C-T-G. }
\end{aligned}
$$

$\mathrm{Fe}$ is a reducer of Nitro group with $\mathrm{HCl}$. So are $\mathrm{Sn}$ and $\mathrm{Zn}$.

$\mathrm{Sz}$ is simple. In an island environment, you have a salty mist. The salt produces $\mathrm{HCl}$. The high iron in diet and water produce $\mathrm{Fe}$. Fe reduces the nitro group on the DNA. That is why there is high $\mathrm{NH}_{2}$ in $\mathrm{Sz}$ blood. The Iron replaces the $\mathrm{NH}_{2}$ on the DNA [1].

\section{Calculations}

The rate of $\mathrm{Sz}$ in the general population is $1.1 \%$. The percentage of people in the general population who take LSD is $33.3 \%$.

*Corresponding author: Dr. Paul TE Cusack, 1641 Sandy Point Rd Saint John, NB, E2K 5E8, Canada, 77030, Tel: 506214 3313; E-mail: St-michael@hotmail.com

Received June 02, 2017; Accepted October 13, 2017; Published October 16, 2017

Citation: Cusack PTE (2017) LSD, Caffeine and Cholera: Possible Causes of Schizophrenia. J Mol Genet Med 11: 296 doi:10.4172/1747-0862.1000296

Copyright: () 2017 Cusack PTE. This is an open-access article distributed under the terms of the Creative Commons Attribution License, which permits unrestricted use, distribution, and reproduction in any medium, provided the original author and source are credited 


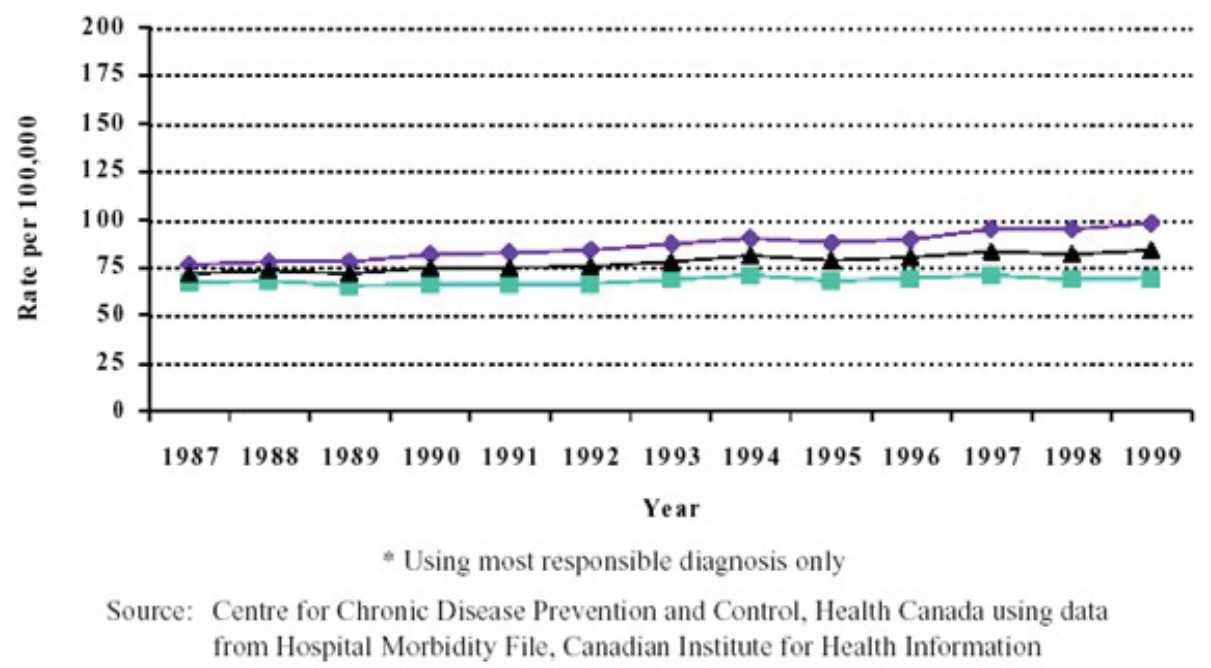

Figure 1: Rates of hospitalization for schizophrenia* in general hospitals by sex, Canada, 1987/88-1999/2000 (Standard to 1991 Canadian population).

So, $\mathrm{LSD} / \mathrm{Sz}=33.3 \% / 1.1 \%=27.3 \sim 28 \%=$ Rise in hospitalization of young men with $S z$.

(The other $0.7 \%$ may be explained by the natural rate of mental illness in the general population due to other causes 90/ $30000=0.3 \%+$ Surviving Parent exposed to Cholera $=1103 /(1103$ $5000) / 30,000=12.99 / 1000=1.3 \%$ ) [3].

The combination of Sz and LSD could be the sole reason for the marked increase in hospitalization for Sz. Those in the age group of 2029 , would have been born in the late 1960's and were adolescents in the 1970 's when LSD was prevalent. I suggest that this is the likely cause for the rate of increase in $\mathrm{Sz}$ among young men.

\section{Cholera and Schizophrenia}

Further to this author's work Schizophrenia [4,5], I hereby provide the possible chemical reaction that shows that Adenine is disrupted by watery diarrhea from the infectious disease of Cholera. It would appear that cholera does damage to that DNA molecule, which leads to a genetic cause for Schizophrenia. Here is the reaction:

Adenine: $\mathrm{C}_{39} \mathrm{H}_{53} \mathrm{~N}_{90} 14 \mathrm{~S}$ (Adenine) $+\mathrm{Fe}+++($ Hyperferremic) +27 $\mathrm{OH}-($ alcoholism $)+3 \mathrm{HCl}$ (acne) $+40 \mathrm{O}_{2}$ (Marine Envt) $\rightarrow 9 \mathrm{NH}_{3}$ (Odorous Urine) $+39 \mathrm{CO}_{2}$ (mosquito attraction) $+\mathrm{SO}_{2}+\mathrm{FeCl}_{3}$ (Ferric Chloride disease $)+27 \mathrm{HOH}$ (Choleric Diarrhea) $+\mathrm{H}_{2}$

\section{Results and Discussion}

Note that all these components are discussed in paper number 4 above. This, then, is the link between cholera and schizophrenia at least and possible other mental illness.

\section{Conclusion}

Ferric Chloride Disease, Hyper Caffeine intake, and LSD were considered to explain the marked increase in Sz among young men from 1987-1999. The most probable cause is use LSD among this group, the results of which we are seeing now. Schizophrenia appears to be linked to cholera which affects adenine. Further studies on this theory are warranted. In addition, further to previous papers on possible causes of Schizophrenia, we provide the chemical calculations indicating that cholera may be the culprit leading to Schizophrenia.

\section{References}

1. Cusack $P$ (2017) Schizophrenia and its causes: Ferric chloride (Cusack's) disease. Men Health Fam Med 2: 1-2.

2. Surveillance and Epidemiology Division (2015) Report Summary - Mental illness in Canada, 2015. Public Health Agency of Canada.

3. Cusack P (2017) Mental illness and cholera. Men Health Fam Med 2: 1-2.

4. Cusack $P$ (2017) More on the robust solution for epidemiology: Nineteenthcentury in Quebec. J Biomet Biostat 8: 1-2.

5. Cusack $P$ (2017) Cholera, iron and mental illness in nineteenth century. Saint John, NB. J Biomet Biostat 8: 1-2. 